\title{
THE CREATION OF HAUMEA'S COLLISIONAL FAMILY
}

\author{
Hilke E. SChlichting ${ }^{1}$ and Re'em SARI ${ }^{1,2}$ \\ ${ }^{1}$ California Institute of Technology, MC 130-33, Pasadena, CA 91125, USA; hes@ astro.caltech.edu, sari@tapir.caltech.edu \\ ${ }^{2}$ Racah Institute of Physics, Hebrew University, Jerusalem 91904, Israel, \\ Received 2009 April 14; accepted 2009 May 22; published 2009 July 13
}

\begin{abstract}
Recently, the first collisional family was discovered in the Kuiper Belt. The parent body of this family, Haumea, is one of the largest objects in the Kuiper Belt and is orbited by two satellites. It has been proposed that the Haumea family was created from dispersed fragments that resulted from a giant impact. This proposed origin of the Haumea family is however in conflict with the observed velocity dispersion between the family members $\left(\sim 140 \mathrm{~m} \mathrm{~s}^{-1}\right)$ which is significantly less than the escape velocity from Haumea's surface $\left(\sim 900 \mathrm{~m} \mathrm{~s}^{-1}\right)$. In this paper we propose a different formation scenario for Haumea's collisional family. In our scenario the family members are ejected while in orbit around Haumea. This scenario, therefore, naturally gives rise to a lower velocity dispersion among the family members than expected from direct ejection from Haumea's surface. In our scenario Haumea's giant impact forms a single moon that tidally evolves outward until it suffers a destructive collision from which the family is created. We show that this formation scenario yields a velocity dispersion of $\sim 190 \mathrm{~m} \mathrm{~s}^{-1}$ among the family members which is in good agreement with the observations. We discuss an alternative scenario that consists of the formation and tidal evolution of several satellites that are ejected by collisions with unbound Kuiper Belt objects. However, the formation of the Haumea family in this latter way is difficult to reconcile with the large abundance of Kuiper Belt binaries. We, therefore, favor forming the family by a destructive collision of a single moon of Haumea. The probability for Haumea's initial giant impact in today's Kuiper Belt is less than $10^{-3}$. In our scenario, however, Haumea's giant impact can occur before the excitation of the Kuiper Belt and the ejection of the family members afterward. This has the advantage that one can preserve the dynamical coherence of the family and explain Haumea's original giant impact, which is several orders of magnitude more likely to have occurred in the primordial dynamically cold Kuiper Belt compared to the dynamically excited Kuiper Belt today.
\end{abstract}

Key words: comets: general - Kuiper Belt - minor planets, asteroids - solar system: formation

\section{INTRODUCTION}

Collisions are thought to have played a major role in the Kuiper Belt ever since its formation (e.g., Davis \& Farinella 1997; Stern \& Colwell 1997; Kenyon \& Luu 1999; Goldreich et al. 2002; Pan \& Sari 2005). This idea is supported further by the recent discovery of the first collisional family in the Kuiper Belt (Brown et al. 2007). Haumea (also known as 2003EL 61 ), one of the largest Kuiper Belt objects (KBOs), is thought to have undergone a giant impact that gave rise to Haumea's rapid rotation with a spin period of only $4 \mathrm{hr}$ (Rabinowitz et al. 2006) and that created its multiple satellite system (Brown et al. 2005, 2006a) and collisional family (Brown et al. 2007). The family of KBOs $\left(1995 \mathrm{SM}_{55}, 1996 \mathrm{TO}_{66}, 1999 \mathrm{OY}_{3}\right.$, $2002 \mathrm{TX}_{300}, 2003 \mathrm{OP}_{32}, 2003 \mathrm{UZ}_{117}, 2005 \mathrm{CB}_{79}, 2005 \mathrm{RR}_{43}$ ) was linked to Haumea because its members display surface properties and orbits similar to those of Haumea. It has been proposed that this family of KBOs are collisional fragments of the ejected ice mantel of Haumea which were produced and ejected in Haumea's giant impact (Brown et al. 2007). However, the velocity dispersion between the family members is only $\sim 140 \mathrm{~m} \mathrm{~s}^{-1}$ which is unusually small for fragments of disruptive impacts which should typically be ejected with a velocity comparable to the escape velocity (i.e., $\sim 900 \mathrm{~m} \mathrm{~s}^{-1}$ for Haumea; Benz \& Asphaug 1999; Nesvorný et al. 2006). In addition, simulations suggest that high-velocity giant impacts lead to either the formation of disk of satellites or the dispersion of collisional fragments. The simultaneous creation of multiple satellites and the dispersion of collisional fragments has not been seen (Melosh \& Ryan 1997; Benz \& Asphaug 1999; Agnor \& Asphaug 2004; Canup 2004, 2005); one should bear in mind, however, that none of the simulations tried to specifically explain Haumea's giant impact.

In this paper, we propose and discuss a different formation scenario for the origin of Haumea's collisional family. In our scenario the family members are ejected while in orbit around Haumea. Ejecting the family members while in orbit around Haumea has the advantage that it naturally gives rise to a lower velocity dispersion among the family members than a direct ejection of fragments from Haumea's surface and, in addition, it aids in explaining Haumea's initial giant impact.

This paper is structured as follows. In Section 2, we introduce our definitions and assumptions. We give a detailed account of our model for the formation of Haumea's collisional family in Section 3. Section 4 is concerned with Haumea's giant impact. Discussion and conclusions follow in Section 5.

\section{DEFINITIONS AND ASSUMPTIONS}

The Haumea family currently consists of Haumea and eight additional KBOs. The family members have typically an eccentricity of $\sim 0.12$ and an inclination of $\sim 27^{\circ}$. The actual masses of the family members are uncertain since Haumea is the only object in its family with a measured albedo. Haumea's visible albedo is with about $70 \%$ (Rabinowitz et al. 2006; Stansberry et al. 2008) among the highest in the solar system. In addition, lower limits for the visible geometric albedo of family members $2002 \mathrm{TX}_{300}, 1995 \mathrm{SM}_{55}$, and $1996 \mathrm{TO}_{66}$ were determined to be 19\% (Ortiz et al. 2004; Grundy et al. 2005), 6.7\%, and 3.3\% (Altenhoff et al. 2004; Grundy et al. 2005), respectively. Given the common origin of the Haumea family and their similar surface characteristics with strong water ice absorption features it is likely that the family members have, like Haumea, high 
Table 1

Definition of Symbols

\begin{tabular}{lcc}
\hline \hline Symbol & Value & Definition \\
\hline$M$ & $4.2 \times 10^{24} \mathrm{~g}$ & Mass of Haumea (Brown et al. 2005) \\
$R$ & $694 \mathrm{~km}^{-1}$ & Mean radius of Haumea \\
$\Omega_{B}$ & $9.2 \times 10^{-4} \mathrm{rad} \mathrm{s}^{-1}$ & Angular breakup velocity of Haumea \\
$v_{B}$ & $635 \mathrm{~m} \mathrm{~s}^{-1}$ & Breakup velocity of Haumea \\
$\Omega$ & $7.1 \times 10^{-10} \mathrm{rad} \mathrm{s}^{-1}$ & Angular velocity around the sun \\
$v_{\text {disp }}$ & $3 \mathrm{~km} \mathrm{~s}^{-1}$ & Velocity dispersion in the scattered Kuiper Belt \\
$\Sigma$ & $3 \times 10^{-4} \mathrm{~g} \mathrm{~cm}^{-2}$ & Kuiper Belt mass surface density of $\sim 100 \mathrm{~km}^{\mathrm{a}}$ sized bodies \\
$m_{S}$ & $\sim 2 \times 10^{20}-3 \times 10^{22} \mathrm{~g}$ & Mass range of Haumea's family member \\
\hline
\end{tabular}

Notes.

${ }^{\text {a }}$ For a density of $3 \mathrm{~g} \mathrm{~cm}^{-3}$ (Rabinowitz et al. 2006).

${ }^{\mathrm{b}}$ Evaluated at $43 \mathrm{AU}$.

${ }^{\mathrm{c}}$ Derived from the magnitude difference between Haumea and the family members, and assuming the same albedo as Haumea and a density of $1 \mathrm{~g} \mathrm{~cm}^{-3}$, magnitudes are taken from Ragozzine \& Brown (2007) and references therein.

albedo surfaces (Rabinowitz et al. 2008). We will, therefore, assume that all family members have an albedo similar to that of Haumea and we calculate the masses of the family members from absolute magnitudes from Ragozzine \& Brown (2007) and references therein.

Estimates from current Kuiper Belt surveys yield for the mass surface density $\Sigma \sim 3 \times 10^{-4} \mathrm{~g} \mathrm{~cm}^{-2}$ for KBOs of $\sim 100 \mathrm{~km}$ in size (Petit et al. 2008; Fuentes \& Holman 2008; Fraser et al. 2008; Trujillo \& Brown 2003; Trujillo et al. 2001). We use this value of $\Sigma$, assuming that no $100 \mathrm{~km}$ sized objects were lost from the Kuiper Belt after it was dynamically excited. We use a power-law distribution $N(r) \propto r^{1-q}$ with power-law index $q \sim 4$ (Bernstein et al. 2004; Fuentes et al. 2009; Fraser \& Kavelaars 2009) when estimating the number density of objects smaller than $\sim 100 \mathrm{~km}$ in this paper.

For simplicity, we define symbols and their numerical values that will be used throughout this paper in Table 1 .

\section{THE FORMATION OF HAUMEA'S COLLISIONAL FAMILY}

\subsection{Formation of a Single Satellite and Ejection by Destructive Satellite Collision}

Our formation scenario for Haumea's collisional family can be divided into three steps. First, Haumea suffers a large collision. This collision gives rise to Haumea's fast, $4 \mathrm{hr}$ spin period and ejects material that accumulates into a tightly bound satellite around Haumea. Second, the newly formed satellite undergoes tidal evolution that increases its orbital separation from Haumea. Third, the satellite suffers a destructive collision with an unbound $\mathrm{KBO}$ which creates and ejects the collisional family (see Figure 1). In this case, the typical velocity dispersion of the family will be of the order of the escape velocity from the satellite which is $\sim 190 \mathrm{~m} \mathrm{~s}^{-1}$ as will be shown below.

Starting with a tightly bound satellites around Haumea, the tidal evolution timescale for a satellite of mass, $m_{s}$, to evolve from an initial separation of a few times Haumea's radius, $R$, to a separation $a \gg R$ is given by

$$
\tau_{\text {tidal }}=\left(\frac{2}{39}\right)\left(\frac{Q}{k}\right)\left(\frac{M}{m_{s}}\right)\left(\frac{v_{B}}{v(a)}\right)^{13} \Omega_{B}^{-1},
$$

where $v(a)$ is the orbital velocity of the satellite with semimajor axis, $a$. $Q$ is the tidal dissipation function and $k$ the tidal Love number of Haumea. We refer the reader to Table 1 for the definitions of the remaining symbols.

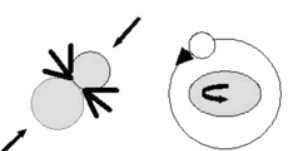

(a)

(b)

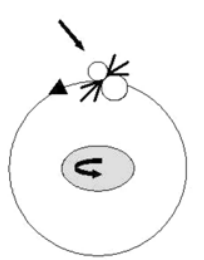

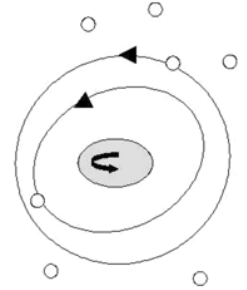

(d)
Figure 1. Illustration of our model for the formation of Haumea's collisional family. From left to right: first, Haumea suffers a giant impact (a). This collision gives rise to Haumea's fast, $4 \mathrm{hr}$ spin period and ejects material that accumulates into a tightly bound satellite around Haumea (b). The newly formed satellite undergoes tidal evolution that increases its orbital separation from Haumea. Haumea's satellite suffers a destructive collision with an unbound $\mathrm{KBO}$ (c). This collision creates and ejects the family and forms the two moons (d).

The satellite suffers a destructive collision with unbound KBOs at a rate

$$
\mathcal{R}_{\text {coll }} \sim \frac{\Sigma \Omega}{\rho r^{3}} r_{s}^{2},
$$

where $r$ is the radius of the "bullet" that can break up the satellite by collisions. The initial satellite needs to have been at least as large as all the identified family members combined (including Hi'iaka and Namaka but with the exclusion of Haumea) which yields a satellite radius of $\sim 260 \mathrm{~km}$. Bodies of this size are predominantly held together by their own gravity. We can estimate the bullet size needed for satellite breakup by considering energy and momentum conservation of the shock that propagates from the impact point to the antipode of the target. Since energy and momentum conservation represent two limiting cases for impact processes (Holsapple 1994), we estimate the range of bullet sizes needed for satellite breakup to be

$$
\left(\frac{G \rho r_{s}^{5}}{v_{\mathrm{disp}}^{2}}\right)^{1 / 3}<r<\left(\frac{G \rho r_{s}^{8}}{v_{\mathrm{disp}}^{2}}\right)^{1 / 6},
$$

where the lower limit is derived by requiring that the kinetic energy of the bullet be equal to the total gravitational energy of the target and the upper limit results from conservation of momentum. We refer the reader to Pan \& Sari (2005) for a detailed derivation and discussion of these two destruction criteria for strengthless bodies. Evaluating Equation (3) yields bullet sizes of $20 \mathrm{~km}<r<70 \mathrm{~km}$. Substituting expression (3) into Equation (2), we find that the typical timescale for Haumea's satellite to suffer a destructive collision is $2 \mathrm{Gyr}<\tau_{\text {coll }}<$ 
80 Gyr. The timescale for satellite breakup by a collision and the consequential formation of the family therefore ranges from a few to tens of Gyr. The actual $\tau_{\text {coll }}$ is most likely closer to the tens of Gyr since the majority of the binaries in the Kuiper Belt should have been destroyed by collisions otherwise. This, therefore, implies a probability for a Haumea-type family forming event of $\sim 10 \%$ over the age of the solar system. Estimates of the current Kuiper Belt population indicate that there should be about 10 objects with radii as larger as Haumea (Trujillo et al. 2001; Bernstein et al. 2004; Fuentes et al. 2009; Fraser $\&$ Kavelaars 2009). The abundance of Haumea-sized objects and the ubiquity of collisionally formed satellites around the largest KBOs (Brown et al. 2006b; Brown \& Suer 2007) makes our formation scenario consistent with having one collisional family Haumea-sized objects in the Kuiper Belt. However, we expect additional collisional families that are associated with smaller parent bodies. For example, we estimate that there are about 300 bodies in the Kuiper Belt with sizes similar to that of the initial Haumea satellite. Given our estimated satellite destruction probability of about $10 \%$, we expect, to an order of magnitude, about $\sim 30$ collisional families that originated from $\sim 260 \mathrm{~km}$ parent bodies. These families, however, would be harder to find without a larger Haumea-type object and might lack any unique spectral signatures that led to the identification of the Haumea family. The typical velocity dispersion between the family members that are produced in the satellite breakup discussed above is of the order of the escape velocity of the initial satellite. For a satellite radius of $\sim 260 \mathrm{~km}$ and a density of $1 \mathrm{~g} \mathrm{~cm}^{-3}$ we have an escape velocity from the satellite, $v_{\mathrm{esc}}$, of $\sim 190 \mathrm{~m} \mathrm{~s}^{-1}$. Simulations of disruptive impacts on ice and basalt targets find typical ejection velocities of $\sim 0.7 v_{\text {esc }}$ for the largest remnant and impact velocities of $3 \mathrm{~km} \mathrm{~s}^{-1}$ (Benz \& Asphaug 1999). It could therefore be that the actual velocity dispersion of the family from a disruptive impact is somewhat smaller than the $\sim 190 \mathrm{~m} \mathrm{~s}^{-1}$ estimated here.

Finally one needs to compare the escape velocity from the satellite, $v_{\text {esc }}$, with its orbital velocity around Haumea, $v(a)$. A satellite breakup only leads to ejection from the Haumea system, and therefore to the formation of a collisional family, if the tidal evolution has increased the orbital separation of the satellite such that $v_{\text {esc }} \gtrsim v(a)$. We need to estimate the tidal Love number, $k$, for Haumea in order to evaluate the orbital evolution timescale. We infer from Haumea's density of $\sim 3 \mathrm{~g} \mathrm{~cm}^{-3}$ that it must be mainly composed of rock. Using the rigidity of basalt rock, $\mu \sim 2 \times 10^{11} \mathrm{erg} \mathrm{cm}^{-3}$ (Benz \& Asphaug 1999), we find $k=1.5 /(1+\tilde{\mu}) \sim 0.01$ where $\tilde{\mu}$ is the effective rigidity given by $\tilde{\mu}=57 \mu /\left(8 \pi \rho^{2} G R^{2}\right)$. Evaluating the tidal evolution timescale in Equation (1) we find that the timescale for the semimajor axis to increase such that $v(a) \sim v_{\mathrm{esc}}$ is $\sim 6(Q / 100)(0.01 / k)$ Myr. The satellite therefore has sufficient time to undergo tidal evolution that increases its semimajor axis such that $v_{\mathrm{esc}} \gtrsim v(a)$ before it suffers a destructive collision. Due to the long collision timescale, the satellite will most likely undergo tidal evolution for $\sim 1$ Gyr before it is broken apart. This yields a satellite separation from Haumea at the time of the satellite breakup of $\sim 17,000 \mathrm{~km}$.

Haumea's spin angular momentum provides an upper limit on the mass of the initial satellite that was later broken up into the family members. Assuming no angular momentum was added to the system after the giant impact and that Haumea was initially spinning close to breakup, we find a maximum satellite orbital angular momentum of $\sim 4 \times 10^{36} \mathrm{~g} \mathrm{~cm}^{2} \mathrm{~s}^{-1}$. For a destructive satellite collision at an orbital separation of $\sim 17,000 \mathrm{~km}$ this yields a satellite mass of $\sim 2 \times 10^{23} \mathrm{~g}$. Our formation scenario, therefore, predicts that the total mass of all the family members combined should not exceed $\sim 1 / 20$ of the mass of Haumea or about three times the mass of the $R \sim 260 \mathrm{~km}$ satellite considered in the calculation for the family forming event above.

In summary, we propose that Haumea suffered a giant impact that leads to the formation of a large, $\sim 260 \mathrm{~km}$ radius, satellite. Tidal evolution increases the semimajor axis of the satellite such that $v_{\text {esc }} \gtrsim v(a)$ in $\sim 10^{7} \mathrm{yr}$. The satellite suffers a destructive collision with an unbound KBO. This collision breaks the satellite into the different family members and ejects them from the Haumea system. This results in a typical velocity dispersion among the family members of $\sim 190 \mathrm{~m} \mathrm{~s}^{-1}$. We propose that Hi'iaka and Namaka are remnants of this collision that did not escape from the Haumea system. The destructive satellite collision that leads to the formation of the Haumea family has a collision timescale of several tens of Gyr which makes our formation scenario consistent with having one collisional family for Haumea-sized objects in the Kuiper Belt.

\subsection{Formation of Multiple Satellites and Ejection by Collisions with Unbound KBOs}

One can imagine that Haumea's initial giant impact did not generate just one but several tightly bound satellites. The newly formed satellites undergo tidal evolution that increases their orbital separation from Haumea. Once the orbital separation is sufficiently large, the majority of the satellites become gravitationally unbound from Haumea due to collisions with small, unbound KBOs. In this case, the typical velocity dispersion of the family will be of the order of the orbital velocity around Haumea before ejection which we show is $\sim 120 \mathrm{~m} \mathrm{~s}^{-1}$.

The rate at which a given satellite suffers collisions with unbound KBOs is given by Equation (2). The satellite sizes and impactor size needed to eject a satellite by collision however differ from that required for satellite destruction in the previous section. In a given collision, the velocity change of the satellite is given by the conservation of linear momentum, $\Delta v(a) r_{s}^{3}=\chi r^{3} v_{\text {disp. }}$. The coefficient $\chi$ accounts for the final momentum of the impactor. If the unbound $\mathrm{KBO}$ is perfectly reflected $\chi=2$. Momentum loss from an impact crater can lead to $\chi>2$ where the exact value of $\chi$ depends on the properties of the colliding bodies (Melosh et al. 1994). Since we are primarily concerned with deriving an order of magnitude estimate for the impactor size we will adopt $\chi=1$ for the rest of this paper. A satellite of Haumea can be ejected by a collision with an unbound $\mathrm{KBO}$ if it suffers a velocity change $\Delta v(a) \sim v(a)$. Therefore, in order to be ejected, a satellite needs to collide with a KBO that typically has a radius of $r \sim r_{s}\left(v(a) / v_{\text {disp }}\right)^{1 / 3}$.

Substituting this expression for $r$ into Equation (2) we have found that the ejection timescale for Haumea's satellites by a collision with an unbound $\mathrm{KBO}$ is given by

$$
\tau_{\text {coll }}=\mathcal{R}_{\text {coll }}^{-1} \sim \frac{\rho r_{s}}{\Sigma}\left(\frac{v(a)}{v_{\text {disp }}}\right) \Omega^{-1} .
$$

For the ejection of Haumea's satellites by collisions with unbound KBOs to be the typical outcome we need $\tau_{\text {coll }} \sim \tau_{\text {tidal }}$ since otherwise most of the satellites should have remained bound to Haumea which is contradicted by observations of the Haumea family. Equating the tidal evolution and ejection timescales allows us to derive the typical velocity with which the 
family members left the Haumea system. Equating Equations (1) and (4) and solving for $v(a)$ we have

$$
\begin{aligned}
v(a) \sim & v_{B}^{13 / 14} v_{\text {disp }}^{1 / 14}\left[\left(\frac{2}{39}\right)\left(\frac{Q}{k}\right)\left(\frac{M}{m_{s}}\right)\right. \\
& \left.\times\left(\frac{\Sigma}{\rho r_{s}}\right)\left(\frac{\Omega}{\Omega_{B}}\right)\right]^{1 / 14} \sim 120 \mathrm{~m} \mathrm{~s}^{-1},
\end{aligned}
$$

where we used $Q / k \sim 10^{4}$ and $m_{s} \sim 4.2 \times 10^{21}$ g (i.e., $M / m_{s} \sim 10^{3}$ ) to estimate $v(a)$. Evaluating Equation (5) for the various masses of the family members we find that $v(a)$ ranges from $98 \mathrm{~m} \mathrm{~s}^{-1}$ to $178 \mathrm{~m} \mathrm{~s}^{-1}$ with a typical value of $\sim 120 \mathrm{~m} \mathrm{~s}^{-1}$. Therefore, Haumea's satellites will be ejected from the Haumea system by collisions with unbound KBOs once their orbital velocity around Haumea is $\sim 120 \mathrm{~m} \mathrm{~s}^{-1}$. This will also be roughly the expected velocity dispersion between family members which is in good agreement with the observations (Brown et al. 2007; Ragozzine \& Brown 2007).

Evaluating both the collisional and tidal evolution timescale using $v(a) \sim 120 \mathrm{~m} \mathrm{~s}^{-1}$ we find from Equations (4) and (1) that the typical ejection timescale is $\sim 60 \mathrm{Gyr}$. The ejection timescales for the various masses of the family members are all tens of Gyr and therefore exceed the age of the solar system. Our calculation here can only estimate the ejection timescale to an order of magnitude, it might therefore be that the actual ejection timescale is somewhat shorter than estimated here. In addition, we might have underestimated the number of unbound KBOs that can lead to the ejection of family members since we extrapolated the surface density of $100 \mathrm{~km}$ sized bodies to smaller sizes assuming a power-law index $q$ of 4 whereas the actual power-law index might be a little larger than this. Since this second formation scenario for the Haumea family involves the ejection of all the family members separately the ejection process cannot be a rare event. The ejection timescales therefore need to be shorter than the age of the solar system for this formation scenario to be feasible. This, however, raises a different problem. If the ejection timescales are indeed shorter than the age of the solar system, then most of the binaries in the Kuiper Belt should have been broken apart by the same process. This is in contradiction with the observations and we therefore conclude that formation of the Haumea family by a destructive collision of a single satellite is the preferred scenario.

In addition to problems discussed above, this second scenario faces yet another challenge. If the initial giant impact of Haumea produced several satellites then satellite-satellite interactions need to be taken into account. The timescale for satellite ejection due to satellite-satellite interactions is $\sim\left(M / m_{s}\right)^{2} a / v(a)$ (Goldreich et al. 2004). This timescale is very short (i.e., $\sim 4 \times 10^{3} \mathrm{yr}$ for $M / m_{s} \sim 10^{3}$ and a 10 day satellite orbit). Initially, however, $v(a)>v_{\text {esc }}$ which implies that the satellites tend to collide with each other rather than eject each other from the system. Satellitesatellite collisions may either lead to accretion or breakup. In either case it is questionable whether several satellites can survive and tidally evolve outward such that they could be ejected by collisions with unbound KBOs. Satellite-satellite interactions are therefore yet another reason to favor our first scenario in which a single satellite is created and broken apart.

As an alternative to ejecting the satellites by collisions with unbound KBOs, one can imagine that the satellites could have been removed from the Haumea system by gravitational scattering of passing KBOs. However, in the high-velocity regime discussed here $\left(v_{\text {disp }}>v_{\text {esc }}\right)$, the rate of satellite ejection due the gravitational scattering is much less than that due to direct impacts of unbound KBOs onto the satellites. See Collins
\& Sari (2008) for comparison of collisional and gravitational evolution of binaries.

\section{HAUMEA'S INITIAL GIANT IMPACT}

Brown et al. (2007) estimated that Haumea's radius before its giant impact was $\sim 830 \mathrm{~km}$ and that the impactor was $\sim 500 \mathrm{~km}$ in radius. The timescale for such an impact to occur in today's Kuiper Belt can be found from Equation (2) which yields a collision timescale of $\sim 8 \times 10^{12} \mathrm{yr}$ when evaluated for $R=$ $830 \mathrm{~km}$ and $r=500 \mathrm{~km}$. Such a collision is therefore extremely unlikely but needed if one wants to form and eject the family directly form the giant impact. Levison et al. (2008) propose a giant impact scenario for Haumea that circumvents this low probability by requiring a collision between two scattered disk objects assuming that the scattered disk was 100 times more massive than it is today. In our formation scenario Haumea can suffer its giant impact before the Kuiper Belt is dynamically excited which shortens the collision timescale significantly. The timescale for Haumea's giant impact in the sub-Hill velocity regime is (Goldreich et al. 2004; Schlichting \& Sari 2008)

$$
\tau_{\text {coll }} \sim \frac{\rho r^{3}}{\Sigma \Omega R^{2}} \alpha^{3 / 2} \sim 8 \times 10^{6} \mathrm{yr},
$$

where $\alpha=R / R_{H} \sim 10^{-4}$ and $R_{H}$ is Haumea's Hill radius. Therefore, allowing Haumea's giant impact to occur while the Kuiper Belt was still dynamically cold decreases the giant impact timescale by six orders of magnitude, even without enhancing the mass surface density in the Kuiper Belt above its estimated current value. We, therefore, propose that Haumea's initial giant impact occurred while the velocity dispersion of large KBOs was still in the sub-Hill regime. This is supported by the ubiquity of small, collisionally formed satellites around KBOs, which have radii as large as $1000 \mathrm{~km}$ (Brown et al. 2006b; Brown \& Suer 2007) and the Pluto-Charon system (Weaver et al. 2006) which strongly suggests that sub-Hill KBO velocities prevailed during satellite formation and that collisional satellite formation was common, especially around the largest KBOs. The satellite, which we propose forms in Haumea's giant impact, is initially tightly bound to Haumea and the long tidal evolution timescale ensures that the Haumea satellite system remains intact until after the dynamical excitation of the Kuiper Belt. The family members are created and ejected from the Haumea system only after the dynamical excitation of the Kuiper Belt which ensures the dynamical coherence of the family members. This scenario, therefore, does not face the potential challenge of removing $99 \%$ of the mass in the scattered disk without destroying the dynamical coherence of the family.

\section{DISCUSSION AND CONCLUSION}

We propose a new formation scenario for the Haumea family. In our scenario, Haumea's giant impact forms a single moon that tidally evolves outward until it suffers a destructive collision from which the family is created. The advantage of this scenario is that it naturally gives rise to a lower velocity dispersion among the family members than expected from direct ejection from Haumea's surface. We show that this formation scenario yields a velocity dispersion of $\sim 190 \mathrm{~m} \mathrm{~s}^{-1}$ among the family members. This is in good agreement with the measured dispersion $\sim 140 \mathrm{~m} \mathrm{~s}^{-1}$ in semimajor axis, eccentricity, and inclination of the family members (Brown et al. 2007; Ragozzine $\&$ Brown 2007) which is a lower limit of the actual velocity dispersion since the orbital angles were chosen to minimize the 
velocity dispersion of the family (Ragozzine \& Brown 2007). Our formation scenario yields about one collisional family for Haumea-sized objects in the Kuiper Belt. Ejecting the family members from Haumea's orbit has the additional advantage that it is easy to reconcile with Haumea's initial giant impact. The family must have been ejected from Haumea after the Kuiper Belt was dynamically excited in order to preserve the dynamical coherence of the family. If the family members are dispersed fragments of the giant impact itself then, the giant impact must have occurred after the Kuiper Belt was dynamically excited. Such a giant impact occurs with a probably of less than $10^{-3}$ over the age of the solar system and is, therefore, extremely unlikely in today's Kuiper Belt. Levison et al. (2008) suggest that Haumea's giant impact could be the result of collision between two scattered disk objects during a phase when the scattered disk was 100 times more massive than it is today. In our scenario, Haumea's giant impact can occur before the dynamical excitation of the Kuiper Belt since the giant impact and the ejection of the family are two different events separated in time by at least $\sim 10^{7} \mathrm{yr}$. The timescale for Haumea's giant impact in the sub-Hill velocity regime is $\sim 8 \times 10^{6}$ yr. Observations show that the majority of the largest KBOs have small, collisionally formed satellites (Brown et al. 2006b; Brown \& Suer 2007). Giant impacts that lead to satellite formation around large KBOs were therefore common in the history of the Kuiper Belt and we propose that Haumea's initial giant impact was one of them. Our formation scenario is also in agreement with results from simulations of giant impacts since it only requires the formation of a satellite and not the simultaneous formation of satellites and direct ejection of fragments in a single collision (Melosh \& Ryan 1997; Benz \& Asphaug 1999; Agnor \& Asphaug 2004; Canup 2004, 2005) which is required in the original formation scenario proposed by Brown et al. (2007).

In addition to the family members discussed above, Haumea has also two satellites. Hi'iaka the larger outer satellite $\left(M / m_{s} \sim 200\right)$ has a semimajor axis of $49,500 \mathrm{~km}$ and a free eccentricity of 0.07 (Brown et al. 2005; Ragozzine \& Brown 2009). Namaka, the smaller $\left(M / m_{s} \sim 2000\right)$ inner satellite, has a semimajor axis of $25,700 \mathrm{~km}$, a free eccentricity of 0.21 and its inclination with respect to Hi' iaka is $13^{\circ}$ (Ragozzine \& Brown 2009). Hi'iaka and Namaka display, just like all other family members, strong water-ice absorption features in their infrared spectra (Barkume et al. 2006; Fraser \& Brown 2009). Since this spectral signature seems to be only present among the family members it seems unlikely that Hi'iaka and Namaka were captured; instead they most likely formed together with the other family members. It is unlikely that Hi' iaka and Namaka evolved to their current separation by tides, since the tidal evolution timescales are excessively long. From Equation (1) we have for Hi'iaka $\tau_{\text {tidal }} \sim 4 \times 10^{12} \mathrm{yr}$ and for Namaka $\tau_{\text {tidal }} \sim 6 \times 10^{11} \mathrm{yr}$ where we used again $Q \sim 100$ and $k \sim 0.01$. Both timescales exceed the age of the solar system by more than two orders of magnitude. We suggest that Hi'iaka and Namaka were produced in the same satellite breakup that created the other family members, only that in their case the impulse was not sufficient to escape Haumea but instead it increased their semimajor axis by a factor of $\sim 2$ to their current separation. Such a collision will however also raise the eccentricity to order unity. It is possible that the satellites, especially Hi' iaka, formed by reaccumulation of collisional fragments of the satellite break up. Such a re-accumulation scenario typically leads to more circular satellite orbits. We also note that Hi'iaka's free eccentricity of 0.07 is consistent with dynamical excitations by passing
KBOs (Collins \& Sari 2008). Namaka, which is ten times less massive than Hi'iaka, could be a single collisional fragment of the satellite breakup, hence its large free eccentricity of 0.21 . We, therefore, find that our formation scenario for Haumea's family can account for the large semimajor axis and modest eccentricities of Hi' iaka and Namaka. The $13^{\circ}$ mutual inclination between the two moons remains somewhat of a puzzle, since it is surprisingly high if the moons formed in a disk and tidally evolved outward and it is surprisingly low if the moons formed from fragments of a disruptive satellite collision as suggested in this paper.

We would like to thank Darin Ragozzine, Mike Brown, and Yanqin Wu for valuable discussions. R.S. is a Packard Fellow. This research was partially supported by the ERC.

\section{REFERENCES}

Agnor, C., \& Asphaug, E. 2004, ApJ, 613, L157

Altenhoff, W. J., Bertoldi, F., \& Menten, K. M. 2004, A\&A, 415, 771

Barkume, K. M., Brown, M. E., \& Schaller, E. L. 2006, ApJ, 640, L87 Benz, W., \& Asphaug, E. 1999, Icarus, 142, 5

Bernstein, G. M., Trilling, D. E., Allen, R. L., Brown, M. E., Holman, M., \& Malhotra, R. 2004, AJ, 128, 1364

Brown, M. E., Barkume, K. M., Ragozzine, D., \& Schaller, E. L. 2007, Nature, 446, 294

Brown, M. E., \& Suer, T.-A. 2007, IAU Circ., 8812, 1

Brown, M. E., et al. 2005, ApJ, 632, L45

Brown, M. E. 2006a, ApJ, 639, L43

Brown, M. E., et al. 2006b, ApJ, 639, L43

Canup, R. M. 2004, Icarus, 168, 433

Canup, R. M. 2005, Science, 307, 546

Collins, B. F., \& Sari, R. 2008, AJ, 136, 2552

Davis, D. R., \& Farinella, P. 1997, Icarus, 125, 50

Fraser, W. C., \& Brown, M. E. 2009, ApJ, 695, L1

Fraser, W. C., \& Kavelaars, J. J. 2009, AJ, 137, 72

Fraser, W. C., et al. 2008, Icarus, 195, 827

Fuentes, C. I., George, M. R., \& Holman, M. J. 2009, ApJ, 696, 91

Fuentes, C. I., \& Holman, M. J. 2008, AJ, 136, 83

Goldreich, P., Lithwick, Y., \& Sari, R. 2002, Nature, 420, 643

Goldreich, P., Lithwick, Y., \& Sari, R. 2004, ARA\&A, 42, 549

Grundy, W. M., Noll, K. S., \& Stephens, D. C. 2005, Icarus, 176, 184

Holsapple, K. A. 1994, Planet. Space Sci., 42, 1067

Kenyon, S. J., \& Luu, J. X. 1999, AJ, 118, 1101

Levison, H. F., Morbidelli, A., Vokrouhlický, D., \& Bottke, W. F. 2008, AJ, 136, 1079

Melosh, H. J., Nemchinov, I. V., \& Zetzer, Y. I. 1994, in Hazards Due to Comets and Asteroids, ed. T. Gehrels, M. S. Matthews, \& A. M. Schumann (Tucson, AZ: Univ. Arizona Press), 1111

Melosh, H. J., \& Ryan, E. V. 1997, Icarus, 129, 562

Nesvorný, D., Enke, B. L., Bottke, W. F., Durda, D. D., Asphaug, E., \& Richardson, D. C. 2006, Icarus, 183, 296

Ortiz, J. L., et al. 2004, A\&A, 420, 383

Pan, M., \& Sari, R. 2005, Icarus, 173, 342

Petit, J.-M., Kavelaars, J., Gladman, B., \& Laredo, T. 2008, in Size Distribution of Multikilometer Transneptunian Objects (The Solar System Beyond Neptune), ed. M. A. Barucci et al. (Tucson, AZ: Univ. Arizona Press), 71

Rabinowitz, D. L., Barkume, K., Brown, M. E., Roe, H., Schwartz, M., Tourtellotte, S., \& Trujillo, C. 2006, ApJ, 639, 1238

Rabinowitz, D. L., Schaefer, B. E., Schaefer, M., \& Tourtellotte, S. W. 2008, AJ, 136,1502

Ragozzine, D., \& Brown, M. E. 2007, AJ, 134, 2160

Ragozzine, D., \& Brown, M. E. 2009, AJ, 137, 4766

Schlichting, H. E., \& Sari, R. 2008, ApJ, 673, 1218

Stansberry, J., Grundy, W., Brown, M., Cruikshank, D., Spencer, J., Trilling, D., \& Margot, J.-L. 2008, in Physical Properties of Kuiper Belt and Centaur Objects: Constraints from the Spitzer Space Telescope (The Solar System Beyond Neptune), ed. M. A. Barucci et al. (Tucson, AZ: Univ. Arizona Press), 161

Stern, S. A., \& Colwell, J. E. 1997, ApJ, 490, 879

Trujillo, C. A., \& Brown, M. E. 2003, Earth, Moon, and Planets, 92, 99

Trujillo, C. A., Jewitt, D. C., \& Luu, J. X. 2001, AJ, 122, 457

Weaver, H. A., et al. 2006, Nature, 439, 943 